\title{
TARMED: une analyse de l'efficacité soi-disant basée sur des faits
}

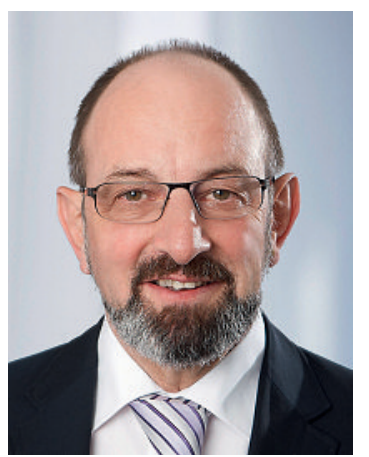

Le TARMED est entré en vigueur le $1^{\text {er }}$ mai 2003 dans le domaine de l'assurance-accidents et le $1^{\text {er }}$ janvier 2004 dans le domaine de l'assurance-maladie. La mise en place d'une structure tarifaire uniforme pour l'ensemble de la Suisse a une longue histoire. Le TARMED devait entre autres améliorer l'indemnisation de la prestation intellectuelle médicale. En 2005, l'EPFZ a présenté une étude exigeant une analyse indépendante et systématique des effets de cette structure tarifaire. Le Contrôle fédéral des finances a répondu à cette recommandation et emploie de grands moyens depuis 2008 pour évaluer le TARMED. Chose étonnante: le secteur hospitalier est totalement mis à l'écart de cette évaluation, bien que $40 \%$ du volume facturé aux assureurs concerne le secteur hospitalier ambulatoire. De plus, cette part a massivement augmenté depuis l'introduction du TARMED.

Le corps médical a participé à cette évaluation en fournissant l'appui professionnel de différentes sociétés de discipline (SSMG, SSMI, SSGO, SSORL, SSO) pour des exemples de cas susceptibles de refléter la réalité des coûts. INFRAS les a analysés de manière approfondie. Après des entretiens avec

\section{L'analyse du TARMED par le Contrôle fédéral des finances est tendancieuse}

les sociétés concernées, INFRAS a rédigé un rapport qui démontre clairement que cinq des onze exemples sont soustarifés. L'évaluation hautement pertinente d'INFRAS éclaircit la question de la réalité des coûts et de l'utilisation correcte du tarif. Ces cinq exemples sous-tarifés représentent en effet plus de $50 \%$ du chiffre d'affaires des cabinets médicaux réalisé avec le TARMED.

Celui qui pensait que le Contrôle fédéral des finances (CDF) recommanderait d'adapter la structure tarifaire en fonction de l'excellent rapport d'INFRAS sera amèrement déçu. En effet, le CDF reste d'avis que la tarification du TARMED offre encore certaines marges de manœuvre et des possibilités d'économiser. Les données et les faits sont là et pourtant le $\mathrm{CDF}$ procède à un choix très sélectif des résultats de cette analyse fouillée et les interprète pour qu'ils étayent les déclarations et les recommandations qu'il souhaite.

Les points faibles indiqués depuis longtemps par le corps médical deviennent ainsi manifestes. La division par deux des cinq dernières minutes de la taxe de consultation, or- donnée par le Surveillant des prix au moment de l'introduction du TARMED, a d'importantes répercussions pour les médecins de premier recours et les défavorise par rapport aux spécialistes. Cela montre clairement que les interventions sur la structure tarifaire influencent uniquement la situation des spécialistes entre eux mais pas l'ensemble des coûts.

\section{Le TARMED désormais dans la compétence de l'OFSP?}

Il est problématique que le CDF (comme aussi les assureurs) mélange constamment interventions structurelles et tarification des prestations résultant de la structure tarifaire. Les positions figurant dans la structure tarifaire repose sur une saisie standardisée des minutages et des coûts ainsi que sur une pondération qualitative des prestations. Une fois introduites dans la structure tarifaire, elles reflètent les rapports corrects entre les prestations dans la structure tarifaire. La tarification n'a lieu qu'après coup par les valeurs du point tarifaire. Alors que la structure tarifaire doit être uniforme au niveau national et qu'elle nécessite l'approbation du Conseil fédéral, l'approbation du tarif de la LAMal se traduit par une convention tarifaire de souveraineté cantonale et n'a rien à voir avec TARMED-Suisse. Les gouvernements cantonaux sont en effet les autorités d'approbation pour les conventions tarifaires LAMal. Les recommandations du CDF atteignent un sommet lorsqu'elles proposent de transférer le TARMED de facto, en tant que tarif officiel, dans la compétence et la responsabilité de la Confédération et donc de l'OFSP. Cela équivaudrait à enterrer la souveraineté cantonale. Nous doutons que cette mesure améliore durablement la situation du corps médical ou qu'elle contribue à baisser les coûts. On se souvient de la révision du tarif de laboratoire!

Dr Ernst Gähler, vice-président de la FMH Responsable du domaine Tarifs et conventions

Un groupe d'experts s'exprimera encore dans l'un des prochains numéros du Bulletin des médecins suisses au sujet de ce rapport et des recommandations du CDF. 